\title{
La transmission intergénérationnelle : Regard sur les services aux particuliers
}

\author{
Samuel GUILLEMOT, Univ Brest, LEGO, Quimper, France
}

\begin{abstract}
Résumé. Recueillir la mémoire intergénérationnelle est devenu une activité économique qui séduit de plus en plus d'entrepreneurs. Pourtant le marché peine à décoller malgré des perspectives de croissance prometteuses. L'objectif de cette recherche est de mieux comprendre le besoin des individus âgés en matière de transmission afin d'adapter les offres de services. A l'appui d'une revue de la littérature et d'une démarche qualitative exploratoire, nous avons mis en évidence plusieurs configurations de transmission (se (re)construire, retrouver un passé idéalisé, faire plaisir à la famille, préserver un patrimoine et s'exprimer). Un des principaux résultats est de montrer que les offres actuelles ne couvrent qu'une partie de ces besoins et négligent les situations de transmission qui s'expriment hors du cadre familial. Nous formulons ensuite des préconisations managériales pour développer ces marchés.
\end{abstract}

Mots-clés. Personnes âgées -Services - Transmission intergénérationnelle

TO CITE: GUILLEMOT S. (2015), LA tRANSMISSION INTERGENERATIONNELLE : REGARD SUR LES SERVICES AUX PARTICULIERS, MANAGEMENT \& AVENIR, 77 (3), 51-69

La question de la transmission intergénérationnelle prend un sens particulier chez les personnes vieillissantes. L'être humain voudrait croire que tout ce qu'il a bâti au cours de sa vie ne va pas disparaître avec lui. Or ces attentes légitimes sont mises à mal par les modes de vie modernes: les familles sont dispersées, les compétences et expériences de nos aînés parfois dévalorisées. Il en découle une souffrance chez les personnes âgées - c'est en tout cas le constat de l'école des grands parents européens ${ }^{1}$. En 2000, Price et al appelaient déjà le marché -marchand ou non - à répondre aux difficultés des individus âgés confrontés à l'éclatement des cadres traditionnels de la transmission. Si bien, qu'aux Etats-Unis, recueillir la mémoire des familles et des individus est devenu une activité économique qui séduit de plus en plus d'entrepreneurs, tant les perspectives de croissance sont prometteuses (Campbell, 2011). L'activité consiste alors à recueillir les souvenirs des personnes vieillissantes et à les figer sur un support qui peut être un livre, un CD ou un DVD. Compte tenu du vieillissement démographique, plusieurs médias économiques américains (Fortune, Wall Street Journal notamment) l'ont placé parmi les «top new business opportunities ». En France, pourtant, le marché peine à décoller. Bien que difficile à quantifier, il existerait entre 800 et 900 prestataires officiellement installés dans le pays. Pourquoi le marché ne se développe-t-il pas? Les offres sont-elles suffisamment adaptées à la demande?

Bien qu'il s'agisse d'un marché niche, la description des services proposés (section 1) et l'élaboration d'un cadre théorique présentant les mécanismes psycho-sociaux sous-tendant ces comportements (section 2) indiquent qu'offrir des moyens adaptés aux personnes âgées pour assouvir leur désir de transmission permet d'augmenter l'estime de soi et de diminuer de l'anxiété face à la mort. Ainsi, une meilleure prise en charge de ces besoins contribuerait au bien-être des individus et de la société dans son ensemble (Ostrom et al, 2010). La transmission étant une pratique hautement symbolique, notre contribution consiste à identifier le sens donné par les individus à leurs pratiques de transmission (section 3 et 4). Cela nous permettra de faire émerger des difficultés et attentes spécifiques (section 5) et ainsi d'élaborer des préconisations managériales concrètes pour développer ces marchés (section 6).

\footnotetext{
${ }^{1}$ EGCP.com
} 


\section{LA STRUCTURE DU MARCHÉ}

Sur le marché générique de la transmission intergénérationnelle, on retrouve plusieurs marchés supports :

$>$ Celui des services biographiques : les prestataires aident les consommateurs à rédiger et mettre en forme l'histoire de leur vie sur un support ;

$>$ Celui des services techniques d'archivage numérique : les prestataires proposent de convertir au format numérique des photographies argentiques, des films $8 \mathrm{mn}$ ou tout autres documents à préserver (par exemple souvenirs-numeriques.com) ;

$>$ Celui des voyages intergénérationnels : quelques agences de voyages proposent des séjours «grands-parents / petits-enfants », le plus souvent autour de thématiques historiques, culturelles et/ou spirituelles. Ainsi «ictus voyages» organise un pèlerinage à Rome permettant aux grands-parents de transmettre à leurs petits-enfants les valeurs du catholicisme ;

$>$ Celui des recherches généalogiques : des généalogistes et héraldistes proposent leurs services pour retracer l'histoire d'une famille ou d'un village (par exemple genealogies-origines.com)

Ces marchés en phase de lancement sont relativement peu structurés et peu concurrentiels, les offres émanant de prestataires isolés. Néanmoins, celui des services biographiques est plus ancien $^{2}$ et commence à se structurer. C'est pourquoi nous allons l'examiner de manière plus approfondie. Tous les acteurs utilisent des arguments liés au devoir de mémoire et à l'importance de la transmission dans la famille pour convaincre le consommateur. Ce qui distingue fondamentalement les offres, c'est la nature des prestataires : indépendants aux moyens limités, sociétés structurées ou association.

\section{L'offre des prestataires indépendants}

Les «biographes privés » sont des personnes physiques - bien souvent auto-entrepreneur et de formation littéraire. Leur métier consiste à interviewer le narrateur selon un guide d'entretien plus ou moins élaboré, enregistrer ses propos pour ensuite les retranscrire et les mettre en forme. Plusieurs séances peuvent être nécessaires pour obtenir la biographie finale. L'histoire de vie ainsi élaborée pourra être livrée sous des formats divers selon la spécialisation du prestataire (papier, CD, DVD). Le plus souvent il s'agit d'un livre agrémenté de photos personnelles édité en un nombre limité d'exemplaires que le consommateur pourra distribuer à ses proches. La prestation est «sur-mesure » et le prix est déterminé en fonction de la nature de la demande (du simple récit d'un évènement marquant jusqu'à la saga familiale sur plusieurs génération). C'est pourquoi, la prestation est généralement facturée à la séance (en moyenne $100 €$ l'heure). Une biographie «standard» revient alors à 2500-3000€. Certains prestataires développent des services complémentaires d'apprentissage des compétences pour les personnes qui souhaitent rédiger seules leur histoire de vie. Ces services peuvent couvrir toutes les étapes de la démarche : des ateliers de réminiscence pour faire revenir les souvenirs, aux ateliers d'écriture pour les mettre en forme jusqu'aux services de correction. Comme nous l'avons souligné en introduction, il est difficile d'évaluer leur nombre exact car certain en font un métier à plein temps, alors que d'autres exercent cette activité en complément d'une autre (écrivain public par exemple).

\footnotetext{
${ }^{2}$ C'est Guillaume Moingeon, biographe breton, qui a «inventé » le métier. Il a débuté son activité en 1996 et a été récompensé en 1999 par plusieurs prix liés à l'innovation et à la créativité entrepreneurial (guillaume.moingeon.com)
} 
Si la flexibilité des indépendants constitue un atout majeur, le manque de moyen en matière de communication (recrutement par bouche à oreille ou via un site internet), la mobilité géographique réduite et l'absence d'harmonisation des prestations et des prix constituent des freins au développement de leur activité. C'est pourquoi, certains indépendants s'appuient sur la force des réseaux à l'image des groupements dans la distribution - par exemple les «compagnons biographes» ou le réseau des «nègres pour inconnus » - avec à la clé partage de compétences, meilleure couverture du territoire, plus de visibilité, et édition d'une charte tarifaire et déontologique pour rassurer le client.

\section{L'offre des sociétés commerciales}

Très peu nombreuses, quelques sociétés avec une marque commerciale (par exemple plume d'éléphant, éditions plumerolles) ont commencé à se positionner sur le marché de la biographie familiale. Si la démarche reste la même (interview, structuration des propos, mise en forme sur un support), le processus est rationnalisé et fragmenté : la société se charge en effet de recruter des interviewers et des écrivains, ainsi que de gérer la relation client. Les offres sont ainsi standardisées, avec des formules «clé en main » ( 2 heures d'entretien pour un livre de 50 pages). Si elles ont l'avantage d'être plus lisibles pour le consommateur, il existe moins de degré de liberté pour le narrateur (grille d'entretien figée, nombre de séances fixé à l'avance). Comme pour les indépendants, certaines sociétés développent également des services complémentaires à destination des personnes qui souhaitent écrire toutes seules : ateliers méthodologiques et/ou services de corrections.

\section{L'offre associative et autres initiatives non marchandes}

Penser la question des services aux personnes âgées conduit à sortir de la seule perspective des services payant et à y inclure les initiatives de soutien et de solidarité non marchandes (Guérin, 2010). Ainsi, des variantes gratuites ou quasi-gratuites assurées le plus souvent par des associations existent en tant que techniques d'animation dans les clubs, associations et maisons de retraités. Souvent articulées autour de thèmes (l'école, les fêtes de village, les premières vacances, etc.), des séances espacées d'intervalles réguliers permettent aux participants de se remémorer les évènements du passé. Ainsi de nombreuses institutions hébergeant des personnes âgées commencent à aider les résidents à écrire, sinon à raconter leur histoire de vie. Le récit de vie y est valorisé comme moyen de communication, vecteur d'échange et de partage car il permet de créer un lien social entre les résidents, entre les résidents et le personnel et parfois même entre les résidents et leurs propres familles (Blanchard, 2006). Ces pratiques ont des vertus thérapeutiques, dans le sens où elles permettent à la personne âgée d'avoir la sensation de contrôle sur sa propre vie à la différence du sentiment d'impuissance ressentie face aux évènements de la vie (Haber, 2006). Ainsi la biographie en tant que technique d'animation ou pratique de soi devient un axe de différenciation et un véritable argument commercial pour les établissements privés (Hartweg et Zehnder, 2003)

La section suivante va mettre en exergue les théories qui permettent de comprendre pourquoi les individus s'engagent dans ces comportements de transmission. 


\section{LES COMPORTEMENTS DE TRANSMISSION INTERGÉNÉRATIONNELLE DES PERSONNES ÂGÉES}

Pour Urien et Guiot (2007), l'anxiété face à la mort et le fait de se rapprocher de l'échéance ultime permettent d'expliquer les efforts réalisés par les individus âgés pour laisser quelque chose à leur image qui va durer au-delà de leur propre vie. Dans cette section, nous allons développer ce point et mettre en exergue les mécanismes psychosociaux mobilisés, ce qui nous permettra de faire émerger les différentes facettes de ces comportements.

\section{Les mécanismes psychosociaux qui sous-tendent les comportements de transmission intergénérationnelle}

Les théories du développement de la personnalité et de l'identité soulignent la multiplicité des sens de soi. Par conséquent, le fait même d'évoquer la préservation et la transmission du self implique, pour les individus, une mise en exergue des aspects du soi qui sont valorisés (via la relecture de vie) pour pouvoir ensuite les préserver et les partager au-delà de leur propre vie (via la générativité).

Construire un sens de soi cohérent. A tout âge, les individus reconstruisent leur passé, évaluent, simplifient et organisent les informations qu'ils possèdent sur eux-mêmes. Ces bricolages identitaires sont nécessaires pour clarifier et cristalliser les dimensions importantes du soi (Webster, 2003). Ainsi la forme narrative convient parfaitement à l'homme moderne, ce qui explique pourquoi l'évolution des écrits biographiques s'est faite en parallèle au processus d'individualisation de la société (Carron, 2002). Dans les dernières années de sa vie, l'individu devient l'historien de sa propre vie: à travers une réflexion et un bilan approfondi, il va tenter de résoudre les conflits internes et reconstruire son propre passé en fonction de l'image qu'il veut laisser (Butler, 1963). Cela explique pourquoi de nombreuses personnes âgées sont engagées dans l'élaboration d'une histoire de vie cohérente qu'elles écrivent «dans leur tête » (de Givenchy, 2004). Cette réflexion approfondie va éclairer des scènes jugées importantes, qui vont alors devenir des moments clés de l'histoire de vie, apporter de nouveaux éclairages sur certains événements de la vie et enfin mettre en valeur des aspects saillants de l'identité (McAdams, 2001).

Préserver et partager ce sens. On parle de générativité, lorsque cette tendance de l'individu à se promouvoir est combinée à un instinct de communion qui pousse les individus à s'abandonner à des choses qui les dépassent (McAdams et StAubin, 1992). Il permet d'expliquer pourquoi certains vont investir une partie d'eux-mêmes (de leur temps, de leurs expériences, voire de leur argent) pour contribuer au bien-être des générations suivantes. A ces forces internes s'ajoute la pression sociale qui encourage à prendre des responsabilités envers les générations suivantes. Celle-ci évolue en fonction de la symbolique des rôles sociaux : parent, enseignant, leader, etc. (Erikson, 1959). Dans ce sens, Vaillant (2002) évoque une phase spécifique à la personne âgée, pour laquelle les préoccupations génératives concernent essentiellement le maintien, la préservation et la transmission de ce qui est valorisé («keep the meaning »). On peut mobiliser la sociologie maussienne du don pour éclairer ce point. Pour Mauss (1923-1924), le don est un fait social organisé autour de trois obligations : celles de donner, d'accepter et de rendre. La dyade donner-recevoir s'incarne dans l'attente d'une forme de reconnaissance, ou dans la satisfaction d'être utile aux autres. L'obligation de rendre fait alors référence à la dette que l'individu éprouve vis-à vis des générations qui le précèdent, ce qui l'encourage à transmettre à son tour ce qu'il a reçu et 
valorisé. Transmettre est dans ce sens une façon de s'inscrire dans les symboles et les significations véhiculés par la famille et/ou la culture. Cela permet de participer à la construction sociale de la réalité, de lier les générations et de s'inscrire dans quelque chose de plus grand, de plus signifiant, de plus éternel que sa simple vie individuelle (Manheimer, 2004).

\section{Typologie des comportements de transmission intergénérationnelle}

Ces forces internes et sociales font émerger deux axes qui structurent les comportements de transmission intergénérationnelle: le bénéficiaire de l'action (bénéfice pour soi ou bénéfices supposé pour les autres) et le rapport au temps (bénéfice relationnel ou bénéfice à titre posthume).

Pour SOI à bénéfice RELATIONNEL. En lien avec le processus de relecture de vie, les personnes âgées ont tendance à rappeler des compétences qu'elles ne possèdent plus ou à se présenter sous des rôles qu'elles ne jouent plus aujourd'hui (Curasi, Price et Arnould, 2004). Pour soi, cela peut servir de points d'ancrage et de stabilité afin de s'adapter aux pertes d'autonomie liées au vieillissement (Wapner, Demick et Redondo, 1990). D’un point de vue relationnel, à travers des conversations, des actions ou la valorisation de certaines possessions, les personnes âgées expriment ce qui a été important pour elles (Unruh, 1983). Lorsque ces objets ont acquis le statut d' «objet-souvenir » irremplaçable, l'individu considère qu'il a le devoir de les conserver et de les transmettre aux générations futures (Guillard et Pinson, 2012). Là encore les motivations peuvent être égoïstes, par exemple : améliorer l'estime de soi, espérer le pardon de ses péchés, régler ses compte, dire sa vérité, en mémoire d'une personne disparues, etc. (Rubinstein, 2002).

Pour LES AUTRES à bénéfice RELATIONNEL. L'acte de transmission est avant tout une action interpersonnelle ; l'histoire et les valeurs individuelles et familiales sont d'excellents vecteurs de liens intergénérationnels (Goldberg, 2009). Dans un monde qui change si vite, il est fréquent que des personnes d'un certain âge écrivent pour leurs petits-enfants, pour leur parler de leur manière de vivre quand ils avaient leur âge. En évoquant son histoire de vie, le narrateur peut chercher à créer une forme de complicité avec le lecteur. En ceci que l'évocation de ses souvenirs entraîne, chez le lecteur, l'évocation de ses propres souvenirs. De même, les individus âgés planifier la distribution de leurs possessions dans une relation complexe entre la signification associée à l'objet et la personne qui va en bénéficier (Price, Arnould et Curasi, 2000). Dans ce sens, les objets «spéciaux » - qui emprisonnent une partie de la mémoire individuelle et familiale - constituent des cadeaux «parfaits » qui permettent d'être une source pour l'histoire familiale et de renforcer les liens avec le partenaire de l'échange (Stevenson et Kates, 1999).

Pour SOI à bénéfice POSTHUME. D'une manière générale, l'identité d'un défunt est définie par ses expériences pré-mortem parce qu'après la mort, il n'y a pas d'opportunité de négocier une nouvelle identité (Bonsu et Belk, 2003). Une des tactiques les plus couramment utilisées est de trouver des supports tangibles au self. La transmission à la génération suivante passe alors par des supports de réminiscence entretenant le souvenir de soi, de ses actions ou encore de son travail (Price, Arnould et Curasi, 2000). De même, certaines personnes vont accentuer l'aspect de l'identité qu'elles souhaitent mettre en valeur en laissant des documents 
écrits, en rédigeant leur biographie ou encore en trouvant un objet qui servira de « véhicule » à l'histoire familiale et individuelle (Belk, 1988). D'autres vont jusqu'à organiser leurs funérailles, rédiger leur notice nécrologique ou encore enregistrer des messages vidéos qui seront proposés aux proches après leur décès (Carroll et Romano, 2010). Il s'agit ici de souligner la signification symbolique du don qui peut instaurer une relation de pouvoir (Le Gall-Ely, 2013), ici un sentiment de dette et/ou une obligation de mémoire pour «ceux qui restent $\gg$.

Pour LES AUTRES à bénéfice POSTHUME. Enfin, les personnes âgées vieillissent avec en mémoire un monde en voie de disparition qu'elles peuvent chercher à préserver. Par des actions concrètes (bénévolat dans des associations culturelles par exemple) ou des aides matérielles (successions patrimoniales, dons, legs à des institutions collectives, etc.), elles deviennent les garants de notre identité collective (Vaillant, 2002). Le témoignage permet alors d'apporter sa modeste contribution à « la connaissance universelle » et de constituer un patrimoine immatériel pour les générations à venir (Lejeune, 2005).

Les motivations à la transmission sont nombreuses et peuvent se combiner. Cette typologie issue de la littérature doit donc être vue comme une grille de lecture. L'objet des sections suivantes est de la confronter à la réalité.

\section{MÉTHODOLOGIE DE LA RECHERCHE}

La méthodologie mise en place a pour objectif d'identifier le sens que les individus dennent à leurs pratiques afin de faire émerger des difficultés et attentes spécifiques. Compte tenu du marché décrit dans la section 1, nous nous centrons dans le cadre de cette étude sur la transmission via la biographie. Dans une perspective exploratoire, nous privilégions la diversité des profils plutôt que leur représentativité. C'est pourquoi nous avons réalisé 21 entretiens semi-directifs de personnes âgées de 60 à 93 ans (moyenne 75,62 ans) - seuil à partir duquel la saturation sémantique a été atteinte. Le critère de recrutement était d'être retraité. En effet, il s'agit là d'un évènement de vie caractéristique du vieillissement propice au développement des préoccupations liées à la transmission intergénérationnelle (Guillemot, 2010). Nous avons veillé à conserver une certaine homogénéité dans la répartition du genre (9 femmes ; 12 hommes), de l'âge (10 personnes de 75 ans et moins; et 11 de plus de 75 ans) et des situations sociales (par exemple un ancien agriculteur, ouvrier, militaire, prêtre, enseignant, médecin, femme au foyer, etc. Les interviews se sont déroulées au domicile des personnes - en face à face ou par téléphone - et ont été menées sur la base d'un guide d'entretien ouvert. Les thèmes abordés étaient les suivants : 1) la relation au passé et aux souvenirs ; 2) la relation à la génération suivante ; puis 3) les motivations à transmettre et les actions menées dans ce sens. Les entretiens ont duré entre 30mn et $1 \mathrm{~h} 30$. Les propos ont été enregistrés et entièrement retranscrits.

Plusieurs lectures phénoménologiques des retranscriptions ont été réalisées afin d'adopter le point de vue des interviewés, puis nous avons abordé le matériau qualitatif avec pour objectifs d'identifier et de développer des catégories conceptuelles qualifiant les expériences, les interactions et les logiques derrières les comportements (Paillé et Mucchielli, 2008). Ainsi 94 catégories qualifiant les projets biographiques ont été identifiées : 58 concernent le processus qui a conduit à la réalisation de la biographie et 36 les actions entreprises pour le diffuser. Par exemple la catégorie «bien-être procuré par l'écriture » est définie comme l'écriture impulsive qui permet de libérer des tensions internes et «la recherche d'attention » 
qualifie l'envoie de son manuscrit à des éditeurs suite au désintérêt manifeste des enfants. Par recoupement progressifs, nous avons identifié 5 configurations.

\section{RESULTATS : LE SENS DONNE AUX PROJETS BIOGRAPHIQUES PAR LES PERSONNES AGEES}

Nous avons identifié cinq cas de figure dans lesquels le projet biographique revêt des significations différentes: se (re)construire; retrouver un passé idéalisé ; faire plaisir à sa famille ; entretenir un patrimoine, et délivrer un message

\section{Se (re)construire}

Raconter sa vie c'est la (re)construire, c'est tisser des liens entre des événements discontinus pour en faire une histoire qui a un sens pour soi et pour les autres. Si pour certains cela reste du domaine implicite, pour d'autres en revanche la construction de sens constitue une fin en soi. En réalité, deux démarches coexistent : une première prospective où l'individu écrit par fragment (écriture de type journaux intimes) et une seconde où l'identité est construite de manière rétrospective (écriture de type mémoires). Les deux sont indépendantes mais parfois liées lorsque la seconde s'alimente de la première :

La démarche d'écriture par fragment permet de garder des traces mais aussi et surtout de comprendre et d'analyser ses erreurs. Ainsi Simon (60 ans, ancien médecin) s'est mis à écrire suite à un divorce mal vécu. Comme « une évidence », il s'est mis à rédiger " de manière compulsive, d'abord pour soi, pour comprendre, pour guérir». On mobilise ici le pouvoir libérateur de l'écriture ; écrire permet en effet de mettre des mots sur un traumatisme et ainsi de faire baisser la tension interne. D'ailleurs le récit de vie écrit de cette manière peut être assimilé ou construit en parallèle à une psychothérapie (Djamila, 63 ans, ancienne employée).

L'envie de faire un bilan est souvent impulsée par les réminiscences liées à l'âge. Le récit de vie peut être envisagé comme la conclusion d'une vie bien remplie. C'est le cas de Pierre (82 ans, ancien chercheur) qui considère d'ailleurs que c'est « une tâche salutaire pour la mémoire » qui permet «de trouver à travers l'existence, ce qui a été le fil conducteur». Néanmoins, l'inventaire de vie n'est pas forcément réalisé en guise de conclusion mais au contraire comme une étape nécessaire pour continuer à avancer. Ainsi Sylvette (63 ans, ancienne bibliothécaire) qui se dit «envoutée devant la richesse du passé » a eu besoin de « réfléchir à la personne qu'elle est devenue ».

Dans les deux cas, raconter son histoire confère au narrateur une nouvelle maitrise de son existence - c'est le caractère performatif du récit. Les difficultés résident dans la mise en place d'une méthodologie ( «on ne sait pas par où commencer. J'ai essayé par l'enfance, on se dit tiens je pourrais faire ça aussi et on arrête et on ne le finit pas », Pierre), l'acquisition des compétences («C'est dur d'écrire! Ecrire c'est quand j'étais plus jeune, à l'école», Marguerite, 89 ans, ancienne agricultrice), ou entretenir la motivation ( j'ai plein de choses à raconter et tout autant de paresse », Djamila). Comme le dit si bien Marie (78 ans, ancienne enseignante): « il est très difficile d'être son propre guide ».

\section{Retrouver un passé idéalisé}

Pour les personnes qui ont du mal à s'adapter au temps présent, le projet biographique est un moyen de recréer une passé idéalisé, une période où elles ont éprouvé une forme de bonheur. Ainsi Anne (75 ans, ancienne chef d'entreprise) a vécu une période prospère pour 
l'entreprise familiale, une référence dans le monde de la construction navale. L'entreprise n'a pas su s'adapter à la société de consommation et a été liquidée. Anne en parle avec amertume : " nos bateaux étaient solides et fait pour naviguer pas comme ceux de maintenant qui sont fait pour parader au port!». Son récit de vie est un moyen de faire revivre le souvenir de l'entreprise et par la même occasion de rendre hommage à son beau-père (le fondateur) et a ses valeurs. La vente du livre (imprimé et vendu par son fils lors des manifestations nautiques) retraçant la saga de l'entreprise familiale a permis de reconstituer une partie des liens sociaux du passé. C'est avec plaisir qu'Anne évoque sa «famille agrandie » constituée d'anciens clients ayant acheté le livre qui ne manquent pas de lui adresser des marques d'attention : «ils y en a, ils nous envoient des cartes postales quant ils naviguent sur nos bateaux !»Ce besoin de recréer les liens du passée est également exprimé par Maurice (81 ans, ancien militaire) qui aime à se remémorer ses expéditions mais « seulement avec d'ancien militaires » car il estime que «seuls ceux qui ont vécu la même chose que [lui] peuvent comprendre ».

\section{Faire plaisir à la famille}

Le projet biographique peut être envisagé comme un moyen de contribuer au bien-être des membres de sa communauté d'appartenance en étant une source pour l'histoire familiale. Le récit de vie est dans ce cas impulsé par un intérêt explicite des destinataires du récit, comme c'est le cas pour Jeannine (75 ans, ancienne employée) dont la nièce lui conseille régulièrement «de mettre tout ça [ses souvenirs] par écrit ». L'enjeu consiste à construire un pont entre les générations en laissant une emprunte des précédentes sur la nouvelle. Les récits peuvent alors prendre la forme d'un hommage aux parents et grands-parents disparus (Yvette, 60 ans, ancienne enseignante) ou à un parcours de vie, le sien et celui de ses proches (Paul, 92 ans, ancien conseiller municipal). Il peut aussi être l'occasion de transmettre un état d'esprit (Jean, 71 ans, ancien ouvrier), ou encore des leçons de vie et des erreurs à ne pas faire (Jacqueline, 60 ans, ancienne aide-soignante). La notion de temporalité, de l'âge et du temps qui passe jouent ici un rôle important : le projet ne peut se concrétise trop tôt - Jacqueline (60 ans) a encore le temps d'y penser - mais il ne faut pas laisser passer son heure comme Louis (93 ans, ancien prêtre) qui se dit « trop fatigué et trop vieux » pour s'y mettre.

Finalement, c'est le support du récit et les occasions de transmission qu'il suscite qui sont valorisés. Le support permet en effet de marquer le coup et de magnifier les moments de transmission. Simon (60 ans, ancien médecin), par exemple, va attendre les 30 ans de son fils pour lui offrir un livre de souvenirs qui retrace son histoire.

\section{Entretenir un patrimoine}

Le projet biographique peut également être un moyen d'entretenir et de valoriser un patrimoine. La différence avec la situation précédente, c'est que l'intérêt de la famille n'est pas manifeste, voire inexistant. Ainsi Sylvette, en plus de sa propre production littéraire, a hérité de tout un tas de papier de famille - dont les journaux intimes de sa mère et une œuvre de fiction inachevé de son père. De cette accumulation naît un sentiment d'attachement. La valeur symbolique est ici très forte, «c'est [son] trésor, il ne faut absolument pas que cela soit perdu » et même si ses propres enfants ne s'y intéressent pas, elle est certaine qu'un jour, en vieillissant, ils «prendront conscience de la chance d'avoir des traces de leur origine ». La tâche qu'elle s'est assignée s'apparente à celle du conservateur de musée dont les fragments de vie constitueraient les pièces. En effet, sa mission consiste à préserver et valoriser un patrimoine biographique et pour cela il faut l'inventorier, "y mettre de l'ordre, le trier, 
l'expliquer ». La tâche est complexe et il faut tenir compte du caractère lacunaire de la mémoire. Ainsi, Pierre aimerait mettre son histoire en perspective avec celle de ses parents et grands-parents, il a bien récupéré des documents dont certaines lettres mais se désole de ne pas connaitre l'histoire qui va avec «C'est frustrant [...] j'essaie de boucher les trous en discutant avec mes frères et sœurs, ce qui est drôle si je puis dire c'est que personne n'a les mêmes souvenirs !».

\section{Délivrer un message}

Le projet biographique peut enfin être vécu comme un moyen d'exprimer et/ou de revendiquer une partie de son identité. Les souvenirs sont ici mobilisés au service d'une forme d'expression, qu'elle soit artistique, militante ou philosophique :

Le récit de vie est un excellent moyen de laisser libre court à son inspiration artistique. Les expériences personnelles sont alors mises en scène, le style littéraire est travaillé. Ainsi, Claude (63 ans, ancien employé de mairie) s'est inspiré de La métamorphose de Kafka, à travers la transformation lexicale - les mots utilisés au début du récit ne le sont plus à la fin il a voulu montrer que "les bouleversements de l'âme l'ont changé ». Bien souvent la biographie est construite comme une œuvre de fiction mais il s'avère très difficile de se détacher de ses propres expériences ainsi Sylvette «essaie d'aller vers la fiction mais le texte reste nourrit de [ses] histoires » ou encore Marie ne désespère pas de « mailler [sa biographie] en roman ».

La figure militante, quant à elle, mobilise ses propres souvenirs au service d'une cause qui lui tient réellement à cœur. A ce titre, le cas de Djamila est très illustratif. Djamila est issue d'une union religieuse mixte et a subi le racisme au quotidien toute son enfance. L'incident qui a suivi le décès de ses parents a ravivé des tensions internes. En effet, du fait de querelles familiales, ces derniers ne sont pas enterrés dans le même pays. Depuis Djamila se sent investit d'une mission : elle doit témoigner, c'est pour elle « la seule façon de faire avancer les choses », elle espère que son expérience "pourra aider des gens dans la même situation qu'[elle]».

Enfin, le récit autobiographique peut être assimilé à un essai où le narrateur exprime ses opinions et souhaite faire partager ses réflexions personnelles sur l'évolution de la société ou sur tel ou tel évènement. Les auteurs se sentent alors obligés de justifier leur légitimité en montant en quoi leurs avis est digne d'intérêt. Par exemple, Bernard (76 ans, ancien ingénieur) a eu une carrière dans la marine nationale et a "vécu tout un tas de choses intéressantes » et s'en sert pour illustrer et donner son opinion sur les conflits qui ont émaillés la deuxième partie du $\mathrm{XX}^{\text {ème }}$ siècle.

Quel que soit le cas de figure évoqué, les personnes investissent beaucoup d'énergie pour faire connaître leur message, il s'agit d'un véritable «travail » (Bernard) à part entière. Le désir de publication est alors fréquent car il constitue un moyen efficace de diffuser le message, par exemple Claude a envoyé son manuscrit à une vingtaine d'éditeurs sans succès. Le monde de l'édition étant relativement fermé, il a finalement déposé son texte dans des archives publiques un moyen de palier le peu intérêt suscité par son récit, de le légitimer et prédire : «dans 50 ans je ne serai plus là mais mon texte existera encore [...]. Il sera lu un jour [...] et il fera du bruit. ».

\section{DISCUSSION DES RESULTATS ET IDENTIFICATION DES ATTENTES LIEES A LA TRANSMISSION}


La figure 1 permet de confronter nos résultats au cadre théorique. Notons que les individus sont principalement préoccupés par la composante relationnelle de la transmission. Au travers des 5 configurations identifiées, notre étude fait ressortir trois types de besoins : ceux liés à la construction d'un message cohérent (lorsqu'il s'agit de se (re)construire et d'entretenir un patrimoine), ceux liés aux moments et aux supports de transmission (faire plaisir à sa famille, retrouver un passé idéalisé) et enfin les problèmes liés aux destinataires (délivrer un message et entretenir un patrimoine).

Figure 1. Confrontation du cadre théorique et des données empiriques

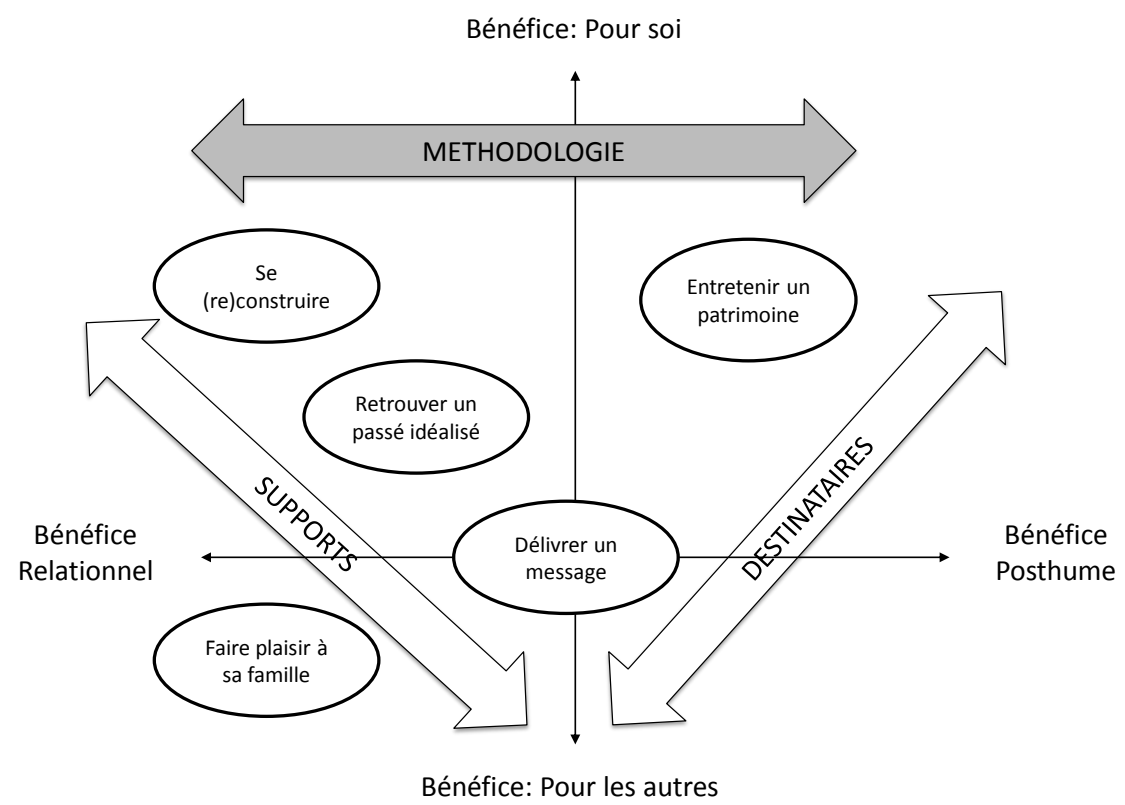

\section{Des supports}

Si nos répondants identifient des moments privilégiés pour transmettre, ils aimeraient bien souvent formaliser et prolonger ces instants précieux. Le support permettrait alors de magnifier et de formaliser ces moments de transmission. Dans ce sens, les services biographiques sont parfaitement adaptés pour ceux qui souhaitent faire plaisir à leur famille car ils permettent de donner un caractère officiel à la transmission et sont un prétexte au partage de photos, d'anecdotes, etc. De plus, le produit fini -sous forme de livre, CD ou DVD - est souvent de bonne facture et permet d'avoir un objet de bonne qualité à transmettre.

\section{Une méthodologie}

Il apparait que la construction d'une histoire cohérente n'est pas une tâche facile. S'il existe un relatif consensus de la communauté scientifique sur l'existence d'une « relecture de vie » (Butler, 1963) dans les dernières années de la vie, le processus est principalement étudié dans un cadre clinique (voir Haber, 2006 pour une synthèse). Hors thérapie, nos données laissent à penser que ce processus interne n'est pas une tâche facile et que les personnes âgées éprouvent des difficultés à unifier et à mettre en valeur les différents aspects de leur identité. Cette difficulté est exacerbée dans nos sociétés postmodernes où l'identité est fragmentée et les objets porteurs de mémoire dispersés : en plus des souvenirs, anecdotes et épisodes de vie à sélectionner et à articuler de manière logique, il ne faut pas négliger les émotions et souvenirs «capturés » dans l'accumulation d'objets, de photographies et autres documents 
(Belk, 1991). Or lorsque les individus souhaitent entretenir un patrimoine, ces éléments ne sont pas forcément dissociés : ils cherchent à articuler leurs propres souvenirs -leur propre biographie-, à la biographie des membres de leur famille à travers les objets porteurs de sens. Si les offres actuelles permettent de préserver la mémoire en articulant de manière logique les souvenirs et anecdotes à raconter, elles négligent complètement la biographie des objets (Kopytoff, 1986) et autres documents à transmettre.

\section{Des destinataires}

Au-delà même du contenu de la transmission et de la construction du message, les individus aimeraient que ceux-ci trouvent une résonance chez les autres. Mais nos données indiquent qu'il est parfois très difficile d'intéresser les destinataires de la transmission (pour ceux qui souhaitent délivrer un message et entretenir un patrimoine). Si la quête de reconnaissance et la revendication des singularités individuelles structurent aujourd'hui la société (Guéguen et Malochet, 2012), l'accélération de l'histoire et de la technologie confèrent une perte d'influence aux aînés. La conséquence de ce double processus apparait dans nos données : nos répondants veulent communiquer mais leur message n'est pas toujours entendu, le(s) receveur(s) peuvent ne pas être réceptif(s) et/ou ne veut(lent) pas entendre. En ce qui concerne la transmission des objets, il existe une solution lorsque personne n'en veut dans la famille : le donner ou le vendre à quelqu'un qui saura l'apprécier (Guillard et Pinson, 2013) mais il n'existe pas d'équivalent pour les histoires de vie. Ce désintérêt engendre chez par nos répondants un sentiment d'injustice, voire un déni de reconnaissance (Honneth, 2006).

\section{PRÉCONISATIONS MANAGÉRIALES}

La discussion qui précède met en évidence que les offres actuelles répondent bien au besoin de supports, qu'elles négligent une partie de la méthodologie et ne pallient pas l' «absence» de destinataires. A partir de ce constat, nous pouvons émettre plusieurs préconisations managériales afin de développer des offres adaptées aux attentes des individus (Figure 2).

Figure 2. Préconisations managériales

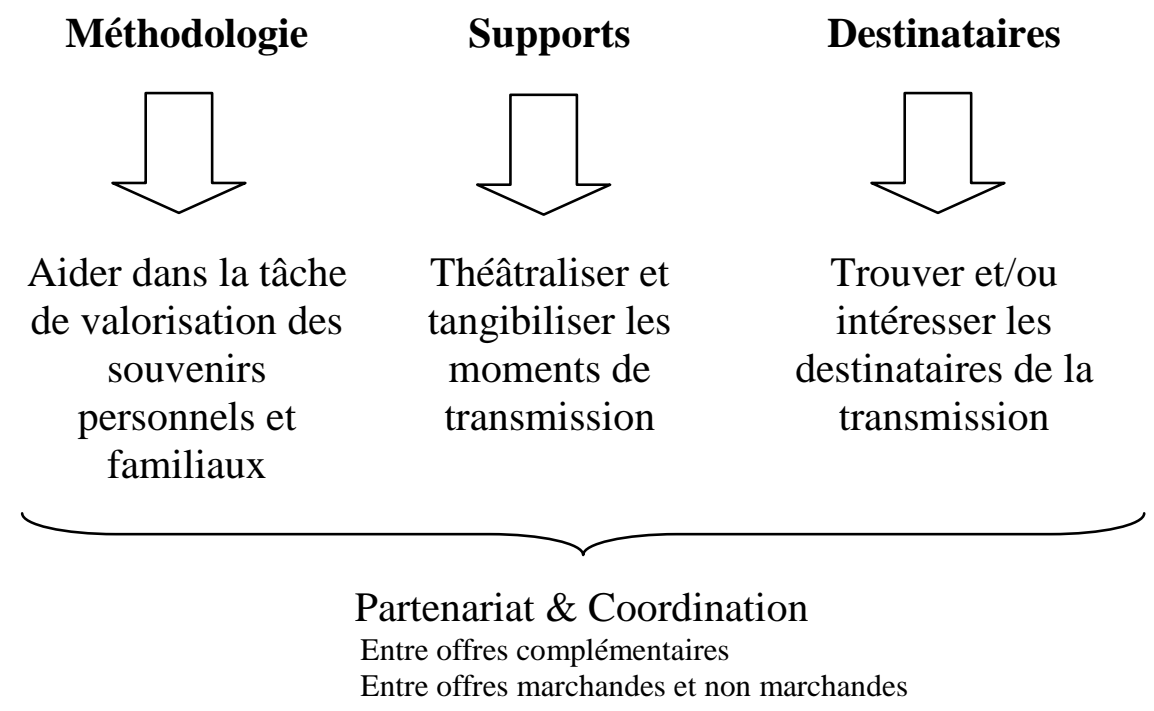




\section{Définir un positionnement clair}

Il s'agit de définir un positionnement clair afin de mettre en évidence des éléments de différenciation qui correspondent aux sens donnés aux projets biographiques :

Aider dans la tâche de valorisation des souvenirs personnels et familiaux. La biographie n'est qu'une façon de transmettre parmi d'autres et ne semble pas se substituer aux autres formes de transmission, dans le sens où elle est complémentaire de la transmission d'autres objets (entretenir un patrimoine) ou d'histoires orales (faire plaisir à sa famille) par exemple. Or les biographes privés se focalisent uniquement sur la valorisation d'un document écrit ou filmé. Nous avons là une piste de développement intéressante pour les prestataires qui pourraient s'inspirer du métier de «personal historian» («historien personnel ») qui se développe aux Etats-Unis : le patrimoine familial (photos, correspondances, objets, etc.) pourrait être utilisé comme «fil narratif», tandis que des recherches généalogiques permettraient de combler les «trous » de l'histoire en vue de mieux la mettre en perspective. A partir de là, on peut penser à des diversifications des offres des biographes vers la restauration des photos anciennes, la conversion de documents tangibles en format numériques (voir section 1). L'idée étant d'aider les consommateurs à construire et entretenir des «musées » personnels et familiaux.

Théâtraliser et tangibiliser les moments de transmission. Les supports de transmission sont valorisés par une partie de la population - notamment dans l'optique de faire plaisir à sa famille et/ou de retrouver un passé idéalisé. Ils permettant de capturer les émotions, formaliser et prolonger les moments de transmission. Il s'agit donc pour les acteurs économiques de poursuivre la logique de tangibilisation des services en concevant des offres permettant aux deux parties (l'émetteur et le(s) destinataire(s)) d'identifier clairement les instants de transmission comme tels. Dans ce sens; les offres - peu nombreuses - des marques commerciales vont dans le bon sens : en développant et en gérant une marque, elles se dotent d'une image et d'une visibilité plus forte; et en entourant l'offre d'indices périphériques tangibles (les packs clés en mains), elles renforcer la « réalité » de leur service. On peut également préconiser d'exploiter les valeurs symboliques des rituels de passage et de progression qui constituent autant d'occasions de se retourner sur l'histoire familiale et propices aux évocations d'un passé commun (Cours et al, 1999). Ainsi on peut imaginer organiser des évènements familiaux avec visionnage de photos ou de films biographiques. En prolongeant cette idée, le «tourisme du souvenir » est un autre moyen de théâtraliser la transmission : il s'agirait d'organiser des voyages sur des lieux de patrimoine chargés de mémoires collectives ou encore vers des villages et musées où le passé serait mis en scène. Un pendant plus individuel peut également être envisagé avec la proposition de circuit personnalisé de « retour » sur les lieux de l'enfance par exemple.

Trouver et/ou intéresser les destinataires de la transmission. Une fois les récits de vie élaborés, les narrateurs ont peu d'opportunités de les faire connaitre en dehors du cercle familial. C'est le cas des individus qui souhaitent «délivrer un message ». Leur démarche s'apparente alors à celles des auteurs «littéraires ». Dans ce sens, les offres biographiques pourraient s'inspirer du secteur de l'édition : en effet les auteurs de romans et de nouvelles ne trouvant pas de débouchés pour leurs œuvres peuvent faire appel à l' «auto-édition » - un service d'impression et de vente à la demande. L'apparition de nouvelles technologies dans le domaine de l'impression à la demande a permis de démocratiser cette pratique. Des sites 
internet proposent aux auteurs de mettre en vente leur livre sur un portail (par exemple the book-edition.com, edilivre.com, lulu.com). Le prix de vente est fixé par l'auteur en tenant compte du prix de fabrication, de la marge du prestataire et des droits d'auteur souhaités. En offrant une existence sociale et un prix (donc une valeur perçue), ces services donnent une légitimé à la production littéraire. Les prestataires de services biographiques doivent donc s'en inspirer en créant leur propre portail de diffusion ou en envisageant des partenariats avec un des sites d'autoédition existant. Il suffirait d'ajouter une rubrique «biographies » ou «témoignages » à côté de celles déjà existantes comme «romans », «nouvelles » ou « poèmes ». Cela permettrait en outre de mettre en valeur les services d'édition (relecture, correction, mise en page, etc.).

D'autres secteurs d'activités peuvent exploiter ces résultats avec comme axe de développement la mise en relation des personnes âgées avec d'autres personnes intéressées par leurs connaissances et compétences. Par exemple, les associations peuvent mobiliser les anciens pour faire découvrir l'histoire d'un quartier ou encore organiser des débats et des rencontres autour de thèmes imposés, etc. On peut également penser à un service d'intermédiation entre les personnes âgées qui ont vécu des évènements spécifiques et les chercheurs (sociologues et historiens notamment) qui étudient ces mêmes évènements. Si cela existe déjà de manière ponctuelle, nos données montrent que cela correspond à une attente réelle et que les offres mériteraient d'être développées.

\section{Développer les partenariats et les réseaux}

Comme suggéré dans les paragraphes précédents, tout en recentrant leurs offres autour de promesses claires les prestataires devraient se diversifier s'ils veulent mieux répondre aux besoins de leurs clients. Le principal frein à ce développement étant le manque de moyens (temporels et financiers pour les indépendants). A l'instar des partenariats horizontaux (entre biographes) décrit dans la section 1, il serait judicieux d'instaurer des partenariats verticaux entre prestataires exerçant des métiers différents mais néanmoins complémentaires dans la chaîne de transmission. Par exemple un partenariat entre un biographe, un site d'autoédition, un généalogiste, un restaurateur de document, un organisateur de voyage, etc. Puis de se présenter sous une interface unique, ce qui suppose un travail de coordination à l'image de ce que font les marques commerciales sur la seule biographie. Il s'agirait de créer un portail de la transmission intergénérationnelle modulable (en 3 volets) selon le souhait des consommateurs. La marque en tant que coordinateur se chargerait de la prospection, de la communication et du recrutement des prestataires. Ces derniers seraient alors déchargés de la partie commerciale et se concentreraient sur leur métier de base, à savoir la partie opérationnelle. La mise en réseau est également un moyen de mettre en commun les moyens notamment de communication - et obtenir plus de visibilité.

Les partenariats peuvent également concerner le marchand et le non marchand. C'est-à-dire que les prestataires peuvent intervenir dans les établissements hébergeant des personnes âgées dans un partenariat gagnant-gagnant. En dispensant des ateliers ou séances d'initiation à la réminiscence et à l'écriture biographie ils permettent d'augmenter le bien-être de la collectivité. Ils peuvent soient contribuer à former les aidants dans une logique d'accompagnement et/ou proposer par la suite des offres plus complètes (et payantes) aux personnes qui désirent aller plus loin dans la démarche biographique.

\section{CONCLUSION}


L'objet de cette recherche était de proposer des pistes de développement applicable à sur un marché émergeant qui peine à se développer alors même qu'il existe un besoin manifeste. En s'intéressant aux significations associées aux pratiques biographiques, notre étude permet de mettre ne valeur plusieurs points qui expliquent pourquoi les services ont du mal à se développer. Tout d'abord les offres actuelles ne concernent qu'une partie de la population. En effet, elles partent du postulat qu'il existe des destinataires identifiés à l'acte de transmission, ce qui n'est pas toujours le cas. Ensuite en se focalisant sur la transmission via un seul support, elles ne répondent pas totalement aux individus qui ne cloisonnent pas autant les médias de transmission (biographie, objets, moments passés ensemble; etc.). Ces constatations nous ont permis de formuler des préconisations afin de restructurer et développer ces marchés.

Cependant, il convient de souligner les limites de la recherche qui constituent autant de voies de recherches futures. Tout d'abord il s'agit d'une étude exploratoire, les profils identifiés doivent faire l'objet d'une nouvelle validation empirique. Une étude quantitative sur un échantillon représentatif de la population permettrait alors de mesurer le marché potentiel. Par ailleurs, les considérations économiques n'ont pas été abordées. En effet, l'usage de ces services n'étant pas encore répandu, nous avons interrogés les individus sur les significations et les motivations associées à leur projet biographique mais pas sur l'utilisation de tels ou tels services. Se pose alors la question de la valeur perçue des services à développer. Il s'agit là d'une question importante qui nécessite des investigations futures. Ensuite, notre étude qualitative est focalisée sur l'avis de l'émetteur du message - la personne âgée -, il conviendrait maintenant de prendre en considération l'avis des «destinataires ». En effet, la transmission est un acte interpersonnel où chacune des parties peut avoir une influence sur le processus. Notons pour terminer, qu'avec l'usage d'internet, les individus laissent désormais autant, sinon plus, de traces numériques que de traces physiques d'eux-mêmes. En effet, nous multiplions les traces de notre identité (blogs, sites personnels, profils de réseaux sociaux, commentaires laissés sur le net, etc.) et une partie des biens potentiellement porteurs de sens (les photographies et les correspondances) tendent à se dématérialiser. Il conviendrait dès lors d'explorer l'impact des technologies numériques sur les comportements de transmission intergénérationnelle.

\section{BIBLIOGRAPHIE}

Belk R.W. (1988), Possessions and the extended self, Journal of Consumer Research, 15, 139-168

Belk R.W. (1991), Possessions and the sense of past, in Belk R.W (coord.) Highways and Buyways: Naturalistic Research from the Consumer Behavior Odyssey, Provo, UT: Association for Consumer Research, 114-130.

Blanchard F. (2006), Rejouer les événements de vie du grand âge, La revue francophone de Gériatrie et de Gérontologie, 123, 128-132

Bonsu S.K. et Belk R.W. (2003), Do not go cheaply into that good night: Death ritual consumption in Asante Ghana, Journal of Consumer Research, 30, 41-55

Butler R.N. (1963), The life review: an interpretation of reminiscence in the age, Psychiatry, $26,65-75$.

Campbell J. (2011), Personal History Business, Self-Counsel Press, Business Series.

Carroll, E. et Romano J. (2010), "Your Digital Afterlife: When Facebook, Flickr and Twitter Are Your Estate, What's Your Legacy?" Berkeley, CA: New Rider's Press

Carron, J-P (2002), Ecriture et Identité, pour une Poétique de l'Autobiographie, Edition Ousia 
Cours D., Heisley D., Wallendorf M. et Johnson F.B. (1999), It's all in the family, but I want it, Advances in Consumer Research, 26, 253-259.

Curasi C.F., Price L.L. et Arnould E.J. (2004), How individuals' cherished possessions become families' inalienable wealth, Journal of Consumer Research, 31, 3, 609-623.

de Givenchy P. (2004), « ma vie est un vrai roman... », In Sudres J-L., Roux G., Laharie M., et de la Fournière F. (coord.), La personne âgée en art-thérapie ; de l'expression au lien social, Paris, L'Harmattan, 93-96.

Erikson E. (1959), Identity and the life cycle: Selected papers, Psychological Issues, 1, 1, 50100.

Goldberg A. (2009), Réaliser une boîte à souvenir, Gérontologie et société, 130, 155-170

Guéguen H. et Malochet G. (2012), Les théories de la reconnaissance, La Découverte, Paris.

Guérin S. (2010), Expérience du don et du care, société de service et personnes âgées, Gérontologie et Société, 135, 167-186

Guillard V. et Pinson C. (2012), Comprendre et identifier les consommateurs qui ont tendance à « tout » garder, Recherche et Applications en Marketing, 27, 3, 57-79

Guillemot S. (2010), Les motivations des personnes âgées au récit de vie et leurs influences sur la consommation de services biographique. Thèse de doctorat en Sciences de Gestion. IAE Brest

Haber D. (2006), Life Review: implantation, theory, research and therapy, International Journal of Aging and Human Development, 63, 2, 153-171.

Hartweg C. et Zehnder G. (2003), Animateurs et animation en établissements pour personnes âgées, Pratiques gérontologiques, Eres édition

Honneth A. (2006), La société du mépris. Vers une nouvelle théorie critique, La Découverte, Paris.

Kopytoff, I. (1986), The cultural biography of things: commoditization as process. In Appadurai A. (Ed), The Social Life of Things: Commodities in Cultural Perspective, Cambridge, Cambridge University Press: 64-91.

Le Gall-Ely M. (2013); Le don dans la recherche en comportement du consommateur et marketing, Recherche et Applications en Marketing, 28, 4, 47-71

Lejeune P. (2005), Signes de vie : Le pacte autobiographique 2, Edition du Seuil, Paris

Manheimer R. (2004), Rope of ashes: Global aging, generativity, and education, In St Aubin E. et McAdams D.P., The Generative Society: Caring for future Generation, Washington D-C, $115-130$

Mauss M (1923-1924), Essai sur le don, Formes et raison de l'échange dans les sociétés archaïques, Paris : Presses Universitaires de France

McAdams D.P. (2001), Unity and purpose in human lives: The emergence of identity as a life story, In Rabin A.I., Zucker R.A., Emmons R.A. et Franck S. (eds), Studying persons and lives, NY: Springer, 148-200

McAdams D.P. et de St. Aubin E. (1992), A Theory of generativity and its assessment through self-report, behavioural acts, and narrative themes in autobiography, Journal of personality and social psychology, 62, 1003-1015

Ostrom, A.L., Bitner M.J., Brown S.W., Burkhard K.A., Goul M., Smith-Daniels V., Demirkan H., et Rabinovich E. (2010), "Moving Forward and Making a Difference: Research Priorities for the Science of Service", Journal of Service Research, 13 (1), 4-36.

Paillé P. et Mucchielli A. (2008), L'analyse qualitative en sciences humaines et sociales, Armand Colin, $2^{\text {nd }}$ édition.

Price L.L., Arnould E.J. et Curasi C.F. (2000), Older consumers' disposition of special possessions, Journal of Consumer Research, 27, 2, 179-201. 
Rubinstein R.I. (2002), Reminiscence, Personal Meaning, Themes, and the "Object Relations" of Older People, In Webster J.D. et Haight B.K. (Eds), Critical Advances in Reminiscence Work : From Theory to Application, Springer Publishing Company, 153-164

Stevenson, G.J. et Kates, S.M. (1999), The last gift: the meanings of gift-giving in the context of dying of AIDS, in Arnould, E.J., Scott, L (Eds), Advances in Consumer Research, Association for Consumer Research, Provo, UT, Vol. 26, pp.113-18

Unruh D. (1983), Death and personal history: strategies of identity preservation, Social Problems, 30, 340-351

Urien B. et Guiot D. (2007), Attitude face à la mort et comportement d'ajustement des consommateurs âgés : Vers l'élaboration d'une réponse marketing, Décisions Marketing, 46, 23-35.

Vaillant G.E. (2002), Aging well, Little, Brown and Company, New-York

Wapner S., Demick J. et Redondo J.P. (1990), Cherished possessions and adaptation of plder people to nursing homes, International Journal of Aging and Human Development, 31, 3, 219-236

Webster J.D. (2003), The reminiscence circumplex and autobiographical memory functions, Memory, 11, 2, 203-215 